ACCEPTED MANUSCRIPT

\title{
Exploring platelet lysate hydrogel-coated suture threads as biofunctional composite living fibers for cell delivery in tissue repair
}

To cite this article before publication: Raquel Costa-Almeida et al 2019 Biomed. Mater. in press https://doi.org/10.1088/1748-605X/ab0de6

\section{Manuscript version: Accepted Manuscript}

Accepted Manuscript is "the version of the article accepted for publication including all changes made as a result of the peer review process, and which may also include the addition to the article by IOP Publishing of a header, an article ID, a cover sheet and/or an 'Accepted

Manuscript' watermark, but excluding any other editing, typesetting or other changes made by IOP Publishing and/or its licensors"

This Accepted Manuscript is @ 2018 IOP Publishing Ltd.

During the embargo period (the 12 month period from the publication of the Version of Record of this article), the Accepted Manuscript is fully protected by copyright and cannot be reused or reposted elsewhere.

As the Version of Record of this article is going to be / has been published on a subscription basis, this Accepted Manuscript is available for reuse under a CC BY-NC-ND 3.0 licence after the 12 month embargo period.

After the embargo period, everyone is permitted to use copy and redistribute this article for non-commercial purposes only, provided that they adhere to all the terms of the licence https://creativecommons.org/licences/by-nc-nd/3.0

Although reasonable endeavours have been taken to obtain all necessary permissions from third parties to include their copyrighted content within this article, their full citation and copyright line may not be present in this Accepted Manuscript version. Before using any content from this article, please refer to the Version of Record on IOPscience once published for full citation and copyright details, as permissions will likely be required. All third party content is fully copyright protected, unless specifically stated otherwise in the figure caption in the Version of Record.

View the article online for updates and enhancements. 
Special issue 'Advances in biomaterials for orthopaedic applications'

\section{Exploring platelet lysate hydrogel-coated suture threads as biofunctional} composite living fibers for cell delivery in tissue repair

Raquel Costa-Almeida, ${ }^{\S, 1,2}$ Isabel Calejo, ${ }^{\S, 1,2}$ Roberta Altieri, ${ }^{3}$ Rui M.A. Domingues, ${ }^{1,2,4}$ Emanuele Giordano, ${ }^{3}$ Rui L. Reis, ${ }^{1,2,4}$ Manuela E. Gomes ${ }^{*}, 1,2,4$

${ }^{1}$ 3B's Research Group, I3Bs - Research Institute on Biomaterials, Biodegradables and Biomimetics, University of Minho, Headquarters of the European Institute of Excellence on Tissue Engineering and Regenerative Medicine, AvePark, Parque de Ciência e Tecnologia, Zona Industrial da Gandra, 4805-017 Barco, Guimarães, Portugal;

${ }^{2}$ ICVS/3B's-PT Government Associate Laboratory, Braga/Guimarães, Portugal;

${ }^{3}$ Department of Electrical, Electronic and Information Engineering "Guglielmo Marconi" (DEI), University of Bologna, Cesena, Italy

${ }^{4}$ The Discoveries Centre for Regenerative and Precision Medicine, Headquarters at University of Minho, Avepark, 4805-017 Barco, Guimarães, Portugal.

$\left[{ }^{\S}\right]$ R. Costa-Almeida and I. Calejo contributed equally to the work.

* Correspondence: Gomes, M.E. (megomes@i3bs.uminho.pt) 


\begin{abstract}
To engineer functional tissue substitutes, it is required a multi-component, multi-scale approach that combines both physical, chemical and biological cues. Fiber-based techniques have been explored in the field of tissue engineering to produce structures recapitulating tissue architecture and mechanical properties. In this work, we engineered biofunctional composite living fibers (CLF) as multi-compartment fibers with a mechanically competent core and a hydrogel layer. For this purpose, commercial silk suture threads were coated with a platelet lysate (PL) hydrogel by first embedding the threads in a thrombin solution and then incubating in PL. The fabrication set-up was optimized and the biological performance was studied by encapsulating human adipose-derived stem cells (hASCs). The developed coating process rendered CLF with a homogenous PL hydrogel layer covering suture threads. Encapsulated hASCs were viable up to 14 days in culture and were able to align at the surface of the core fiber and deposit collagen types I and III.

In summary, the study shows that PL-hASCs hydrogel coated suture threads represent a simple multi-compartment and multifunctional system, with PL hydrogel offering biofunctionality to guide the biological activities of encapsulated cells in addition to the replication of tissue-level mechanical support provided by the suture threads.
\end{abstract}

Keywords: Biofunctional composite living sutures; Cell encapsulation; Cell-laden fibers; Fiber-based techniques; Multi-compartment fibers; Tissue engineering 


\section{Introduction}

Fiber-based techniques and fibrous structures have been widely explored in the field of tissue engineering owing to their potential to emulate the architecture and mechanical properties of different tissues, from cardiac muscle to musculoskeletal tissues, as skeletal muscle, tendons and ligaments [1-5]. Over the past decades, several fabrication methods have been used and refined to produce cell-laden fibers, namely electrospinning [3], wetspinning [6], microfluidics [7], microfluidics spinning [8], microfluidics combined with polyelectrolyte complexation [9, 10]. However, engineering functional tissues frequently requires a multi-component, multi-scale approach to simultaneously fulfill physical, chemical and biological requirements of native tissues. Hence, the concept of composite living fibers (CLFs) has been recently proposed $[11,12]$. These multi-compartment fibers comprise a robust core to replicate physical properties at the tissue-level, providing mechanical support to the engineered or healing tissue. Additionally, an outer hydrogel layer offers a hydrated biomimetic environment adequate for cell encapsulation. Previously developed CLFs have been demonstrated to support cell survival and to withstand textile assembling [11, 12]. One of the most attractive features of CLF is the possibility of tuning the properties of each compartment independently. We have further developed composite suture threads based on CLF biofabrication concept by coating commercial silk sutures with a hydrogel composed of alginate and methacrylated gelatin (ALG:GelMA) [13]. After encapsulating human tendon derived cells (hTDCs), we have demonstrated cell migration and alignment, as well/as up-regulation of genes associated with tenogenesis and matrix remodeling [13]. This previously developed system has shown interesting features, but lacked biofunctionality. Therefore, in the present study, we further introduced a source of human factors with therapeutic value in a cost-effective manner by taking adavantage of the intrinsic biofunctional properties of platelet lysate (PL) as a coating for composite living fibers.

Platelet-rich hemoderivatives (PRHd) have been capturing great attention in the biomedical field as alternative cost-effective sources of biomolecules with therapeutic value [14-16]. Particularly, PL can be obtained from platelet concentrates by the cryogenic disruption of platelets and constitutes a protein-rich cocktail comprising several growth factors (e.g., platelet-derived growth factor (PDGF), transforming growth factor (TGF)- $\beta$, vascular endothelial growth factor (VEGF), basic fibroblast growth factor (bFGF), insulin-like growth factor (IGF), among others) and molecules 
involved in cell adhesion (e.g., fibrin, fibronectin and vitronectin) [14, 15]. PLderived biomaterials, including hydrogels [17-20], scaffolds [21-23] and patches [24, 25], have been receiving great attention toward overcoming some limitations associated to bolus injections of therapeutic growth factors or platelet-derived preparations (e.g., rapid leakage, short half-life of soluble factors, denaturation, injection risks, etc.), constituting a promising strategy for endogenous regenerative medicine.

In this study, we engineered platelet lysate hydrogel-coated suture threads encapsulating human adipose-derived stem cells (hASCs) as prospective biofunctional composite living fibers. Herein, we devised a coating platform to generate PL hydrogel-coated sutures as a way to modify the outer layer of CLF while maintaining the mechanical robustness of core multifilament silk suture threads. We evaluated the effects of different incubation times with clotting factors in inducing PL fibrillation and hydrogel coating formation. To assess the biological performance of PL-hydrogel coated suture threads, hASCs were encapsulated within the PL hydrogel layer. Upon encapsulation, proof-of-concept studies on cellular viability, alignment and matrix deposition were performed over time in culture.

\section{Materials and methods}

\subsection{Materials}

Non-absorbable braided surgical suturing threads (SURGISILK, DW2901R) were purchased from Sutures (Sutures Ltd., UK) and used as received. Calcium chloride $\left(\mathrm{CaCl}_{2}\right)$, bovine thrombin (thrombin from bovine plasma lyophilized powder, 40-300 NIH units/mg protein), Phosphate-Buffered Saline (PBS), glutaraldehyde (Grade I, $25 \%$ in $\mathrm{H}_{2} \mathrm{O}$ ), Triton $\mathrm{X}-100$, Bovine Serum Albumin (BSA), phalloidin solution (Phalloidin-Tetramethylrhodamine B isothiocyanate) and 4,6-Diamino-2-phenyindole dilactate (DAPI, $5 \mu \mathrm{g} / \mu \mathrm{L}$ ) solution were purchased from Sigma-Aldrich (SigmaAldrich Corporation, USA). Minimum Essential Medium alpha ( $\alpha$-MEM), fetal bovine serum (FBS), and an antibiotic/antimycotic solution (AB/AM) were purchased from Life Technologies. A final $\alpha$-MEM solution was prepared with $10 \%(\mathrm{v} / \mathrm{v}) \mathrm{FBS}$ and $1 \% \mathrm{AB} / \mathrm{AM}(\mathrm{v} / \mathrm{v})$. Alamar Blue was purchased from SeroTec (Biorad, Portugal) and formalin (10\% neutral buffered formalin) was purchased from Thermo Scientific (Thermo Fisher Scientific, USA). 


\subsection{Preparation of platelet lysate (PL)}

Platelet lysate (PL) was prepared using platelet concentrates (PC) from healthy human blood donors provided by Hospital de São João (Serviço de Imunohemoterapia do Centro Hospitalar São João, Porto, Portugal) under a cooperation protocol, as previously described [24-26]. Briefly, PC with a platelet count of 1 million platelets $/ \mu \mathrm{L}$ were used. PC were pooled using ten donors and then subjected to three freeze/thaw cycles using liquid nitrogen at $-196{ }^{\circ} \mathrm{C}$ and a $37{ }^{\circ} \mathrm{C}$ water bath, respectively, to promote platelet lysis and protein content release. Platelet debris was removed by centrifugation at $1400 \mathrm{~g}$ for 10 minutes at $4{ }^{\circ} \mathrm{C}$. Supernatants were stored at $-80^{\circ} \mathrm{C}$ and thawed immediately prior to use.

\subsection{Fabrication of PL hydrogel coated sutures}

Suture threads were coated with a hydrogel layer composed of PL. For this purpose, sutures were fixed inside a circular mold and subsequently immersed into thrombin solution prepared in $5 \mathrm{mM} \mathrm{CaCl}_{2}$ at a final concentration of $2 \mathrm{U} / \mathrm{mL}$. A total volume of $1 \mathrm{~mL}$ was used for an approximate length of $20 \mathrm{~cm}$. Different incubation periods were tested $(15,30,45$ and 90 minutes) and sutures were incubated at room temperature. Then, sutures were transferred to a clean channel, which was then filled with equal amounts of fresh PL solution $\left(57.5 \mathrm{mg} \cdot \mathrm{mL}^{-1}\right.$ of total protein). Sutures were incubated in PL at $37^{\circ} \mathrm{C}$ for 2 hours under humidified conditions to allow for PL gelation. Upon hydrogel optimization, 90 minutes incubation in thrombin: $\mathrm{CaCl}_{2}$ solution was selected for subsequent assays. In optimized conditions, after the incubation period, the gelation is complete and the mixture (either PL alone or PLhASCs in the cellular assays) is entirely coating the core thread. The final hydrogel coating is composed of $100 \%$ of PL, which is composed of albumin, growth factors, cytokines and structural proteins (e.g., fibrinogen, vitronectin and fibronectin).

The morphology of PL hydrogel coated sutures was analyzed by optical microscopy (DM750, Leica, Schweiz), in order to observe hydrogel layer formation around suture threads core. Herein, eight images per suture were acquired immediately after the coating process (Day 0) and after 24 hours (Day 1), and the PL hydrogel layer thickness was assessed using Image J software 1.8.0_112 version.

\subsection{Cell culture and encapsulation}


Human lipoaspirate samples were obtained from healthy patients undergoing plastic surgery under informed consent, according to the Declaration of Helsinki and to protocols approved by the Ethical Committee of Hospital da Prelada (Porto, Portugal). Human adipose-derived stem cells (hASCs) were isolated through enzymatic digestion, following previously described protocols [27, 28]. Human ASCs were expanded and cultured in $\alpha$-MEM supplemented with 10\% (v/v) FBS and 1\% $\mathrm{AB} / \mathrm{AM}(\mathrm{v} / \mathrm{v})$ and used at passages 2-4.

For experiments, cells were trypsinized at $80 \%$ confluency and suspended in PL solution using a concentration of $5.5 \times 10^{5}$ cells $\mathrm{mL}^{-1}$. Cell-PL mixture was used to coat suture threads as described above. After encapsulation, PL-hASCs hydrogel-coated suture threads were imaged under an optical microscope and hydrogel thickness was determined overtime.

\subsection{Cell viability by live/dead and Alamar Blue assays}

Live/dead assay: Viability of encapsulated hASCs was determined by fluorescence labeling through live/dead assay. After 1, 3, 7 and 14 days in culture, PL-hASCs hydrogel coated suture threads were removed from culture medium, washed with sterile PBS and then incubated with $1 \mathrm{mg} / \mathrm{mL}$ calcein acetoxymethyl (Invitrogen, 1:500) and $1.5 \mathrm{mM}$ propidium iodide (PI, Invitrogen, 1:1000) solution in PBS for 20 minutes at $37{ }^{\circ} \mathrm{C}$. After, samples were washed with sterile PBS and visualized under a fluorescence mircospose (Imager Z1m, Zeiss).

Alamar Blue assay: The metabolic activity of encapsulated hASCs was evaluated after 1, 3, 7 and 14 days in culture by Alamar Blue assay, according to manufacturer's instructions. At each time point, culture medium was removed, samples were washed with sterile PBS and incubated in $10 \%(\mathrm{v} / \mathrm{v})$ Alamar Blue solution in culture medium at $37{ }^{\circ} \mathrm{C}, 5 \% \mathrm{CO}_{2}$ for 4 hours. The fluorescence of the supernatant was measured using a plate reader (Synergy HT, Bio-Tek Instruments, $560 \mathrm{~nm}$ of excitation and 590 nm of emission). Then, samples were washed twice with sterile PBS and fresh culture medium was added. Data for each individual sample were normalized to day 1. Cellfree fibers were used as controls.

\subsection{Morphological characterization by SEM}


The morphology of PL-hASCs hydrogel coated suture threads was assessed by scanning electron microscopy (SEM). For this purpose, samples were collected after $1,3,7$ and 14 days of culture and fixed with $2.5 \%$ of glutaraldehyde at $4{ }^{\circ} \mathrm{C}$. Then, samples were dehydrated in a series of increasing concentrations of ethanol $(20,30$, 50, 80, 90 and 95\% v/v), being incubated in 100\% ethanol overnight. Air-dried specimens were carefully mounted on sample holders using double-sided carbon adhesive tape and sputter coated with platinum prior to morphological observation by High-Resolution Field Emission SEM (FIB-SEM, Auriga Compact, Zeiss) at an accelerating voltage of $5 \mathrm{kV}$.

\subsection{Immunocytochemistry}

To assess the ability of encapsulated cells to produce ECM proteins, cell-laden fibers were processed for immunofluorescence analysis. After 1, 3, 7 and 14 days of culture, samples were fixed in $10 \%(\mathrm{v} / \mathrm{v})$ neutral buffered formalin solution for 20 minutes at room temperature and permeabilized using a $0.25 \%(\mathrm{v} / \mathrm{v}$, in PBS) Triton X- 100 (Sigma) solution. Non-specific binding was blocked using $1 \%(\mathrm{w} / \mathrm{v}$, in PBS) of bovine serum albumin (BSA, Sigma) and all antibodies were diluted in the blocking solution. Samples were incubated with rabbit polyclonal anti-human collagen I (abcam, ab292, dilution 1:500) or rabbit polyclonal anti-human collagen III (abcam, ab7778, dilution 1:100) for 1 hour at room temperature, being then washed in PBS and subsequently incubated with the secondary antibody Alexafluor 488 donkey antirabbit (Life Technologies, A21206, dilution 1:1000) for 1 hour at room temperature. Finally, F-actin filaments of the cytoskeleton were stained with phalloidin (Phalloidin-Tetramethylrhodamine B isothiocyanate from Amanita phalloides, P1951, Sigma, 1:200 in PBS) for 20 minutes; and nuclei were counterstained with 4,6 Diamidino-2-phenyindole dilactate (DAPI, $5 \mu \mathrm{g} / \mu \mathrm{L}$, D9564, Sigma) for 10 minutes. Negative controls were performed in the absence of the primary antibody. All samples were visualized using a confocal laser scanning microscope (Leica TCS SP8, Microsystems, Wetzlar, Germany).

\subsection{Actin filaments organization by 2D-FFT}

The degree of F-actin alignment on encapsulated hASCs was evaluated by 2D-fast Fourier transform (2D-FFT) using confocal images of phalloidin staining, as previously described [29]. Confocal images from days 3 and 14 were first converted 
to 8-bit grayscale and cropped 1002 - 1002 pixels for 2D-FFT analysis with Image $\mathrm{J}$ software (NIH, USA), supported by oval profile plug-in (http://rsbweb.nih.gov/ij/plugins/oval-profile.html). The radial intensities of the 2DFFT frequency plots were calculated and plotted against the angle of rotation from $0^{\circ}$ to $360^{\circ}$. To compare directly the samples, the exported values of intensity were normalized and plotted in arbitrary units.

\subsection{Morphometric analysis}

Nuclei aspect ratio of encapsulated hASCs was determined after 3, 7 and 14 days in culture by analyzing confocal images of DAPI staining using ImageJ software. Nuclei aspect ratio was determined by measuring and dividing the length by the width of each nucleus. A total of 50 nuclei were measured in each condition using different confocal images.

\subsection{Statistical analyses}

Results were obtained from at least three independent experiments with a minimum of three replicates for each condition. Results are presented as mean \pm standard error of the mean (SEM). Statistical analyses were performed using GraphPad Prism 7.04 version software. One-way and two-way analyses of variance (ANOVA) with posthoc Tukey's and Sidak's multiple comparíson tests were used. A difference between experimental groups was considered significant with a confidence interval of $95 \%$, whenever $p<0.05$.

\section{Results}

\subsection{Fabrication of multi-compartment biofunctional PL hydrogel-coated suture} threads

Commercially available multifilament silk sutures were used as core threads to develop multi-compartment biofunctional composite living fibers. To achieve PL gelation and consequent hydrogel formation, thrombin needs to be added to the system in order to induce fibrinogen self-assembly. Herein, suture threads were first fixed inside a mold and immersed in a solution containing thrombin and calcium ions (Figure 1A-i). Different incubation periods were tested. Afterwards, suture threads were carefully transferred to clean molds and further incubated with PL solution for 2 
hours at $37{ }^{\circ} \mathrm{C}$ inside a customized humidified chamber (Figure 1A-ii), enabling PL matrix polymerization and crosslinking.

A Optimization of PL hydrogel coating

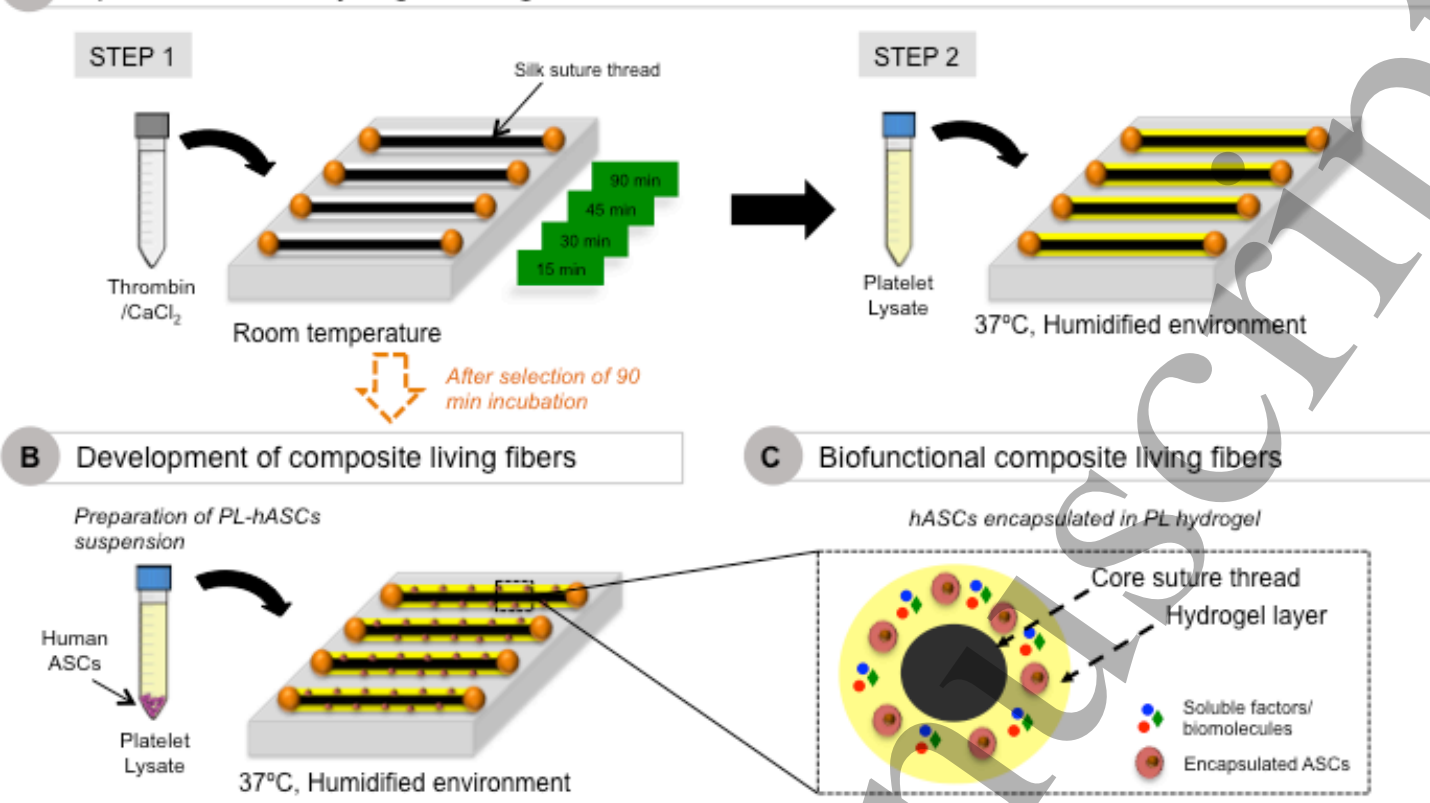

Figure 1. Schematic illustration of the fabrication process of biofunctional composite living fibers. (A) Steps for the optimization of hydrogel coating by incubating silk suture threads for different incubation times in thrombin $/ \mathrm{CaCl}_{2}$ solution and subsequently immersing the threads in PL. Upon optimization and selection of an incubation time, the development of composite living fibers was achieved by first incubating silk suture threads in thrombin/ $\mathrm{CaCl}_{2}$ for 90 minutes and then in (B) a solution of human adipose-derived stem cells (ASCs) suspended in PL solution. (C) Representation of the concept of biofunctional composite living fibers, which comprise a PL hydrogel, as a cocktail of soluble factors with therapeutic potential, encapsulating human ASCs.

Preliminary assessments showed that this incubation period is needed to enable the generation of a homogeneous PL hydrogel layer around the core thread (Figure S1). Figure 2A shows the aspect of PL hydrogel-coated suture threads immediately upon fabrication (Day 0, top) and after 1 day in aqueous environment (PBS solution, Day 1, bottom). Hydrogel thickness was determined at both times (Figure 2B). After fabrication, hydrogel layer thickness varied between $42.7 \pm 5.5 \mu \mathrm{m}$ and $73.5 \pm 9.2$ $\mu \mathrm{m}$. Incubating suture threads for a period of 15 minutes in thrombin/ $/ \mathrm{CaCl}_{2}$ solution resulted in a significantly thinner hydrogel layer $(p<0.0001)$. After 1 day, threads incubated during 15 minutes in thrombin/ $\mathrm{CaCl}_{2}$ had a reduction of approximately 
$20 \%$ in hydrogel layer thickness. Although no differences were found between 30 and 45 minutes of incubation, 1 day after fabrication, these threads exhibited a reduction in hydrogel layer thickness of approximately $13 \%$ and $12 \%$, respectively $(p=0.0331$ for 30 minutes). Threads incubated for 90 minutes in thrombin/ $\mathrm{CaCl}_{2}$ solution resulted in thinner hydrogel layers, in comparison to 45 minutes ( $p=0.0499)$, but no significant reduction in the hydrogel was found (approximately $6 \%$ from day 0 to day 1 ). Hence, this condition was selected for further cell encapsulation studies (Figure 1B, Figure $2 \mathrm{C})$.

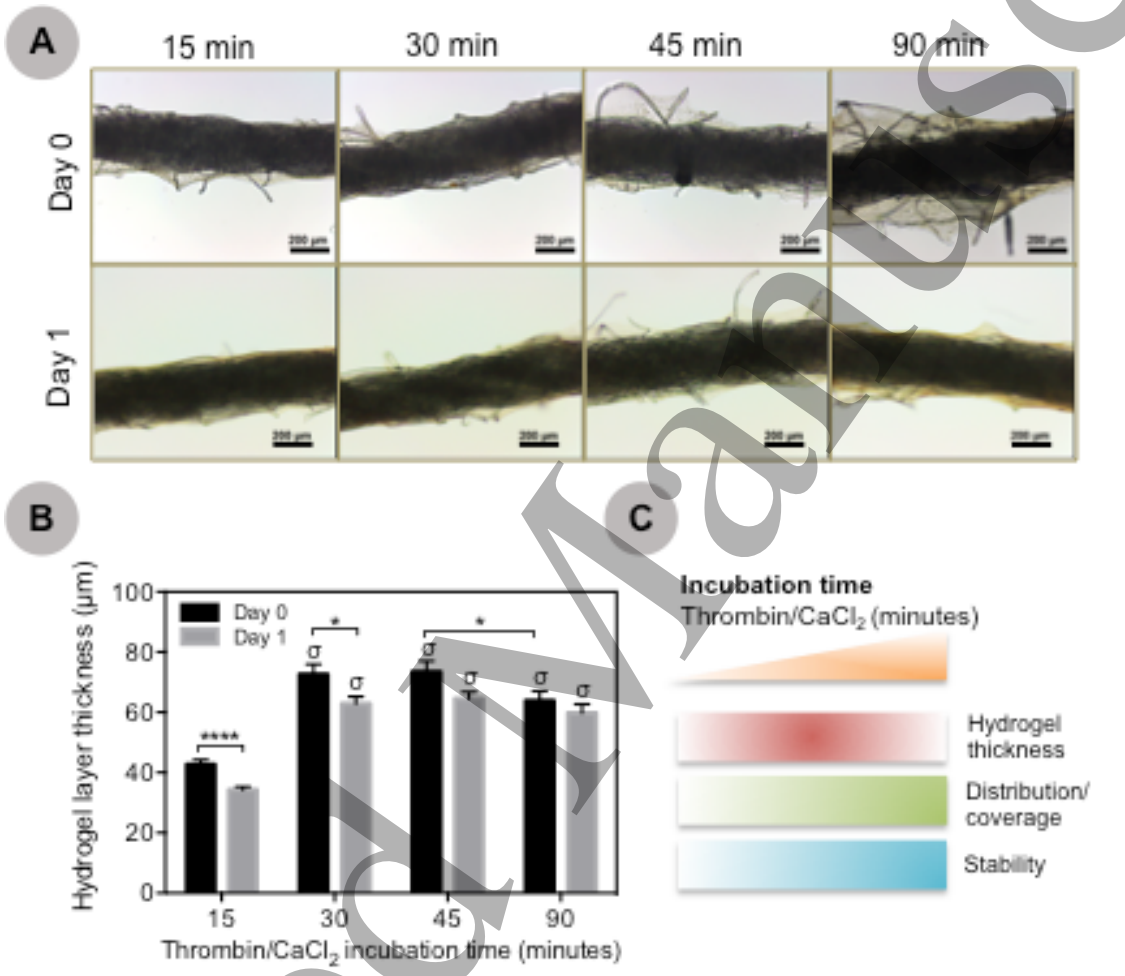

Figure 2. Morphology of multi-compartment biofunctional composite living fibers and hydrogel thickness. (A) Representative optical microscopy images of multi-compartment fibers. Scale bars, $200 \mu \mathrm{m}$. (B) Variation of hydrogel thickness as a function of thrombin $/ \mathrm{CaCl}_{2}$ incubation time. Statistically significant differences are shown as *, $p<0.05$; ****, $p<0.0001$ and $\sigma, p<0.0001$ in comparison to 15 minutes incubation at the correspondent day. (C) Schematic summary of the effects of increasing incubation times with thrombin $/ \mathrm{CaCl}_{2}$ solution in hydrogel layer properties. Distribution/coverage refers to the overall coverage of the silk suture thread with a homogeneous PL hydrogel layer.

\subsection{Cell encapsulation and viability}


The biological activity of the developed system was evaluated by encapsulating hASCs. For this purpose, cells were mixed with PL solution prior to coating. Upon fabrication, a uniform distribution of PL-hASCs hydrogel layer could be achieved around the suture thread (Figure 3A). Immediately after encapsulation, a gross layer of PL hydrogel with round cells could be observed with an average thickness of 45.9 $\pm 1.8 \mu \mathrm{m}$ (Figure 3A, Day 0), but this PL-hASCs hydrogel layer changed overtime (Figure 3A, Day 1 - Day 14). Indeed, hydrogel layer thickness significantly decreased with time in culture (Figure $3 \mathrm{~B} ; p<0.0001$, for all time points in comparison to day 0 ). From day 1 to day 7 (Figure 3B), PL-hASCs hydrogel layer thickness showed a reduction from $30.0 \pm 1.3 \mu \mathrm{m}$ (Day 1) to $21.3 \pm 1.6 \mu \mathrm{m}$ (Day $7 ; p<0.0001$; Day 3 versus Day $7, p=0.0002)$. Strikingly, after 14 days in culture, PL-hASCs hydrogel layer thickness significantly increased $(35.7 \pm 2.3 \mu \mathrm{m} ; p=0.0010$, in comparison to Day $1 ; p<0.0001$, in comparison to Day 3 and Day 7 ), although still thinner than in Day $0(p<0.0001)$.

Cell viability and metabolic activity upon encapsulation were respectively assessed by live/dead staining (Figure 3C) and Alamar Blue assay (Figure 3D). Figure 3C depicts high cellular viability 1 day after hASCs encapsulation and up to 14 days in culture. Furthermore, cellular metabolic activity significantly increased over time in culture (Figure 3D; $p<0.0001$, Day 1/Day 3 versus Day 7 and Day 14). Together, these results indicate that PL hydrogel-coated suture threads are suitable to support cellular growth. 
A

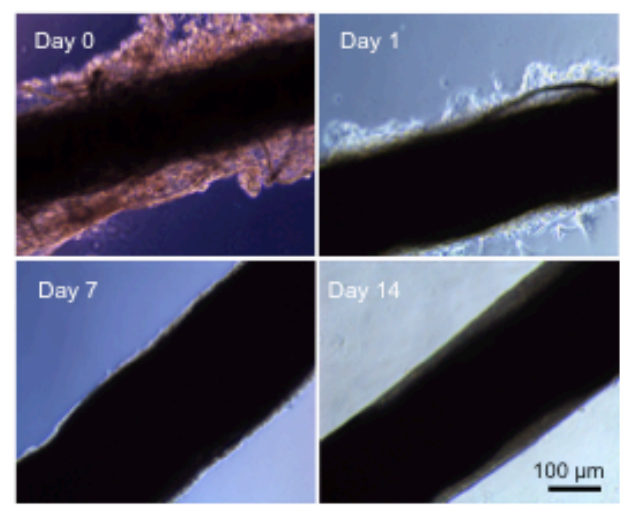

C

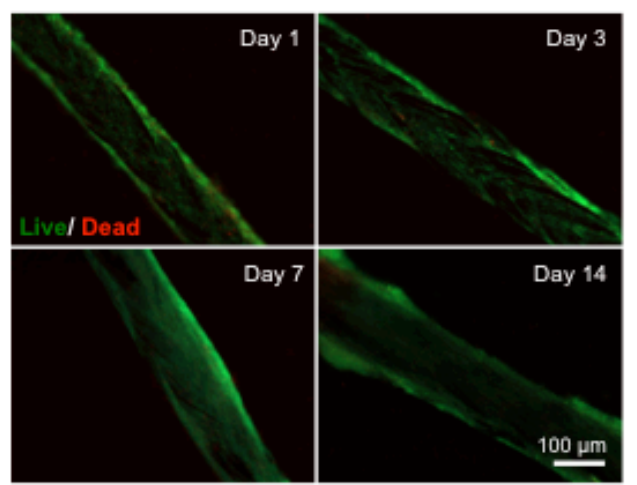

B

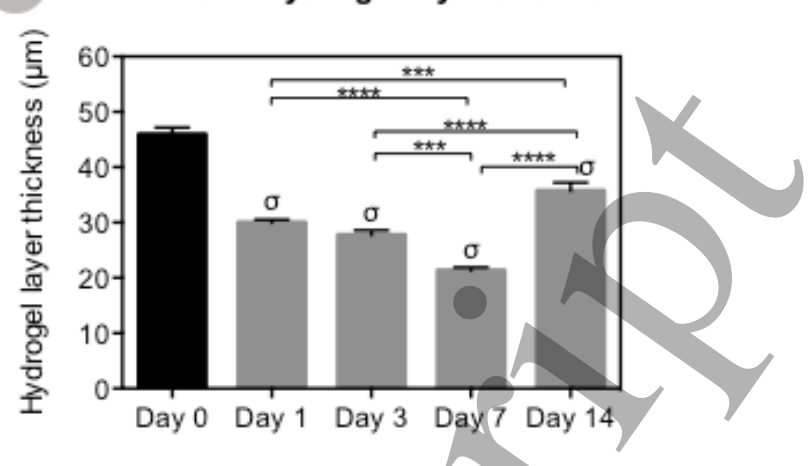

D

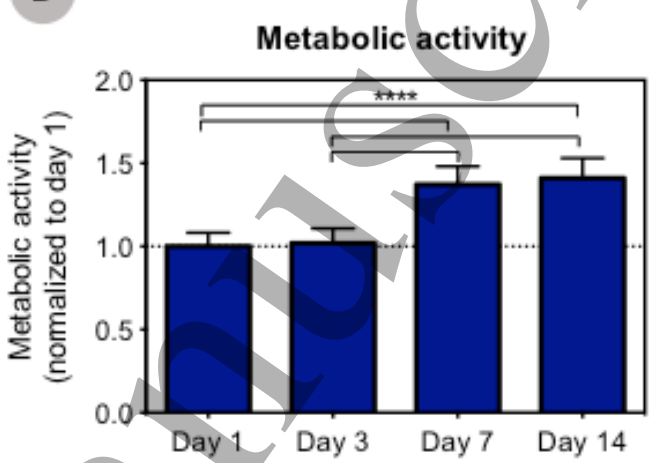

Figure 3. Cell encapsulation and viability. (A) Optical microscope images of PLhASCs composite living fibers immediately after fabrication (day 0 ) and after 1, 7 and 14 days in culture. Scale bar, $100 \mu \mathrm{m}$. (B) Hydrogel layer thickness upon cell encapsulation (PL-hASCs hydrogel layer, day 0) and up to 14 days in culture. Statistically significant differences are shown as $* * *, p<0.01 ; * * * *, p<0.0001$ and $\sigma$, $p<0.0001$ in comparison to Day 0. (C) Fluorescence microscope images of live/dead staining of biofunctional composite living fibers after 1, 3, 7 and 14 days in culture. Live cells are green (calcein AM) and dead cells are red (propidium iodide). Scale bar, $100 \mu \mathrm{m}$. (D) Cellular metabolic activity determined using Alamar Blue assay. Results are normalized with respect to the values for day 1 . Statistically significant differences are shown as $* * * *, p<0.0001$.

\subsection{Cellular organization and alignment}

Distribution and organization of encapsulated hASCs were studied in biofunctional composite living fibers. Again, a homogeneous layer of cells could be observed around the core suture threads over time in culture (Figure 4A). Interestingly, encapsulated hASCs were able to organize in an aligned manner as soon as after 7 days in culture (Figure 4A). Indeed, staining of cytoskeletal actin filaments (Figure 4A, bottom panel) suggests that encapsulated cells could migrate from the hydrogel 
compartment, reaching the surface of the core thread and aligning following the direction of the microfibers composing the suture. The alignment of actin filaments was further assessed by 2D fast Fourier transform (2D-FFT) analysis, comparing Day 3 vs. Day 14 (Figure 4B). Cytoskeleton alignment is evident after 14 days in culture, as seen by the two symmetric sharp peaks (Figure 4B), suggesting anisotropic alignment of encapsulated hASCs, which is less evident at Day 3. Furthermore, nuclei aspect ratio was determined in encapsulated hASCs. As depicted in Figure 4C, a value closer to 1 corresponds to rounded nuclei. The average aspect ratio of cell nuclei significantly increased with time in culture (from $1.4 \pm 0.4$ at Day 3 to $2.4 \pm 0.5$ at Day 14, $p<0.0001$, Figure 4C).
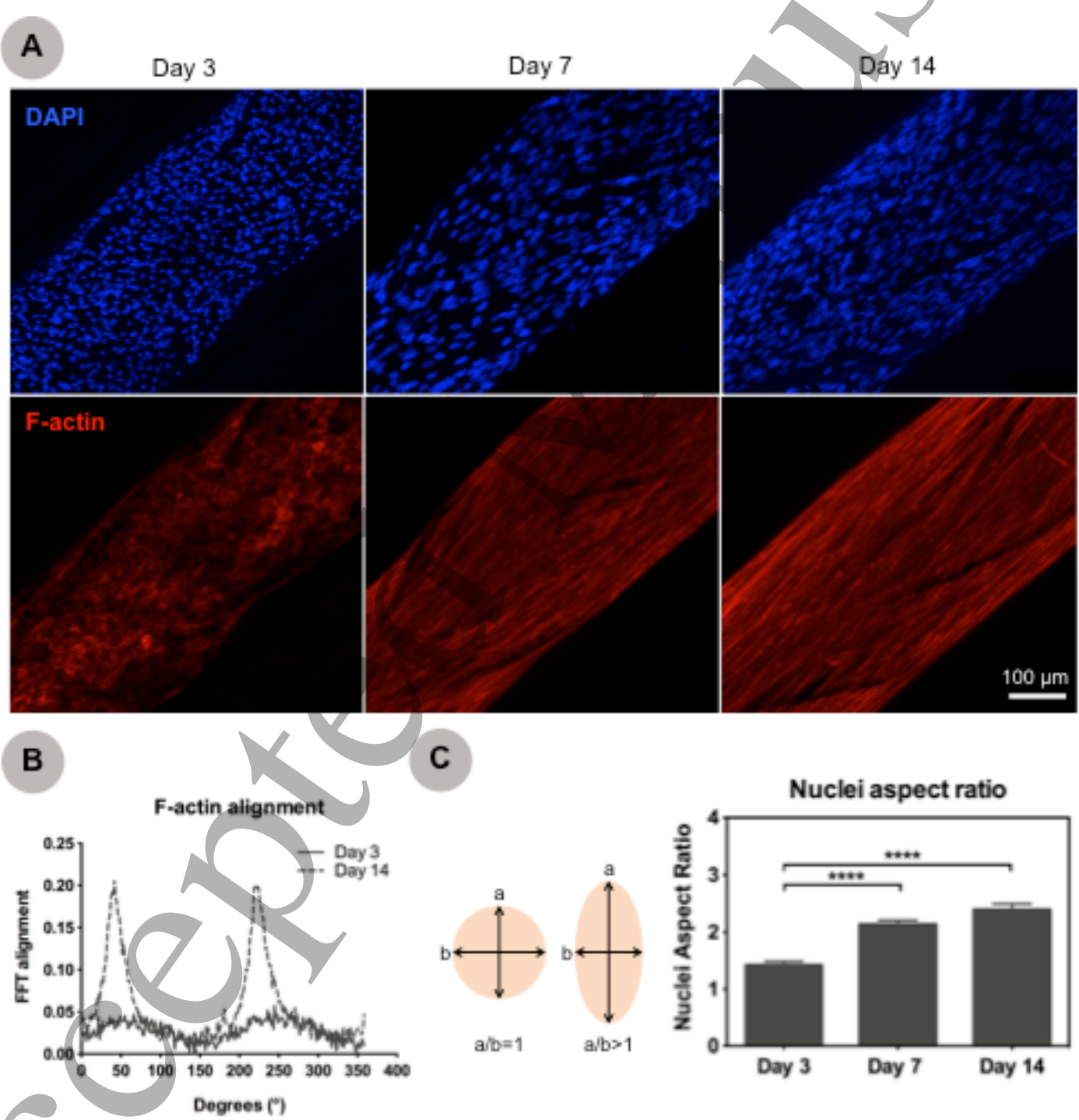

Figure 4. Morphometric analysis of encapsulated hASCs. (A) Confocal images of nuclei (DAPI, blue) and actin filaments (F-actin, red) staining after 3, 7 and 14 days 
in culture upon encapsulation. Scale bar, $100 \mu \mathrm{m}$. (B) 2D-FFT frequency plots comparing actin filaments alignment between day 3 and day 14. (C) Average nuclei aspect ratio (length versus width of nucleus) at days 3, 7 and 14. Statistically significant differences are shown as $* * * *, p<0.0001$.

\subsection{ECM production and organization}

The deposition of newly synthesized ECM components by hASCs encapsulated within PL hydrogel layer was evaluated. Figure 5 shows the morphological changes occurring over time in culture. Indeed, until Day 3, the surface topography of the core suture is clearly seen (Figure 5, bottom panel). From Day 3 to Day 7, this initial topography becomes less evident, suggesting that the outer compartment composed of PL-hASCs hydrogel is being remodeled, which occurs simultaneously with a significant decrease in thickness, as described above. After 14 days in culture, a complete cell-ECM layer covering the suture thread was observed (Figure 5, right panel).

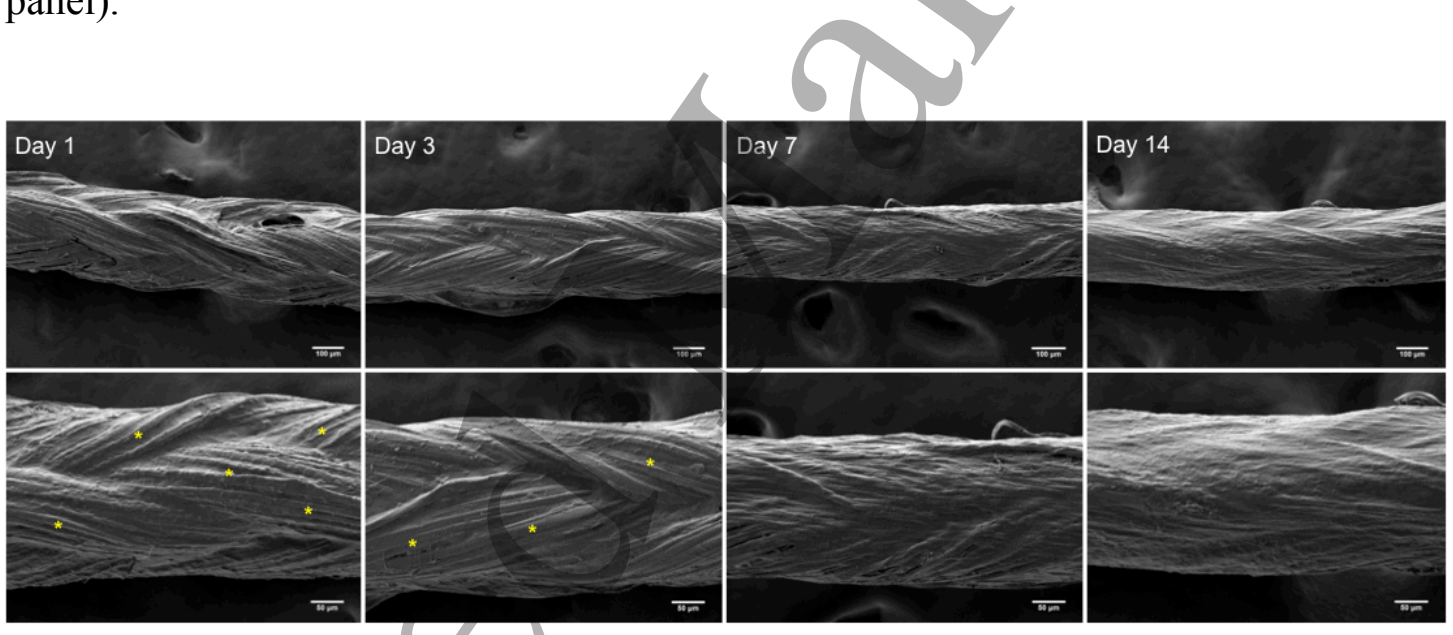

Figure 5. Morphology of biofunctional composite living fibers. SEM images of PL hydrogel coated suture threads after 1, 3, 7 and 14 days in culture. Scale bars, $100 \mu \mathrm{m}$ (top panel) and $50 \mu \mathrm{m}$ (bottom panel, higher magnifications). Stars represent areas in which the original suture can easily be distinguished.

Additionally, the expression of collagenous proteins was investigated. Encapsulated hASCs were found to express both type I and III collagen (Figure 6). Initially, after 7 days both ECM proteins were mainly localized intracellularly (Figure 6, left panel), whereas after 14 days, a notable layer of collagen type I appeared to be organizing following the anisotropic alignment of encapsulated hASCs (Figure 6, top-right 


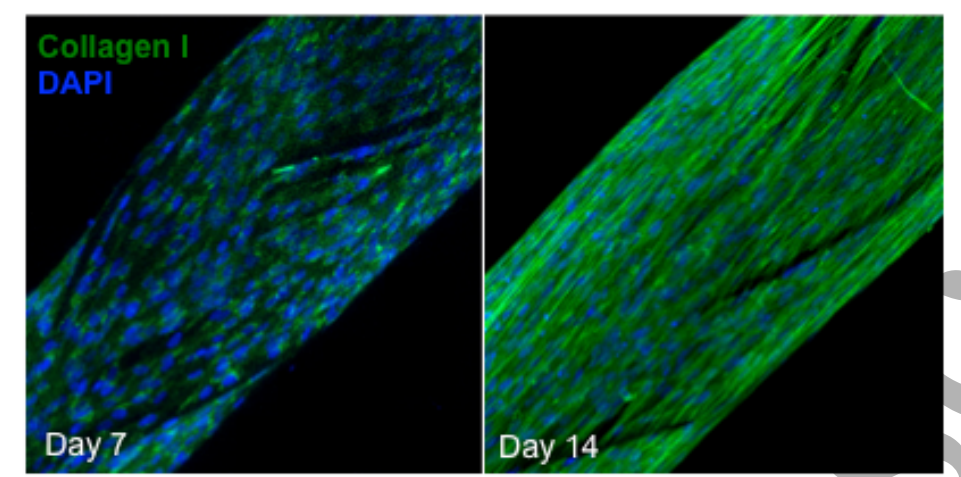

image). These results suggest that the developed biofunctional composite living fibers could support cellular activities of remodeling their surrounding microenvironment, particularly through the production of a collagen-rich matrix.

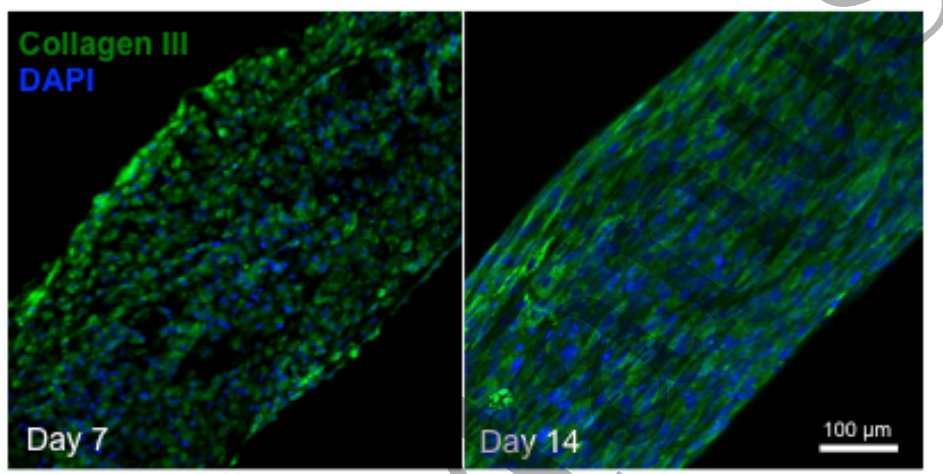

Figure 6. ECM deposition by hASCs in biofunctional composite living fibers. Confocal images of immunostainings against collagen type I (green, top panel) and collagen type III (green, bottom panel) after 7 and 14 days in culture. Nuclei were counterstained with DAPI (blue). Scale bar, $100 \mu \mathrm{m}$.

\section{Discussion}

Over the years, biotechnological progresses have been disrupting biomaterial design strategies. The fields of tissue engineering and regenerative medicine have become strongly dependent on the development of advanced and complex bioengineered materials. Nonetheless, translation of such advances can be limited by difficulties in matching tissue- and cell-level properties simultaneously. Composite living fibers are composed of a mechanically robust core and a hydrated environment that supports and nurtures encapsulated cells, enabling the generation of complex living constructs. Multi-component approaches frequently involve firstly the fabrication of a scaffold, which is then embedded into a hydrogel precursor solution and further crosslinked. Alternatively, CLF allow for a fine control over cell distribution within the final 
construct. Indeed, the intertwining of different cell-laden fibers provides the possibility of precisely positioning cells from the inside outwards of a $3 \mathrm{D}$ construct $[11,13]$, prospectively enabling tissue regeneration to occur within the dimensions of the whole construct. Herein, we explored the concept of composite living fibers using commercially available silk suture threads coated with platelet lysate, a cocktail of therapeutic factors that can be either obtained from the patient or as a commercial GMP conform clinical grade preparation.

As a proof-of-concept study, we developed a coating strategy and further assessed the effects of PL hydrogel-coated suture threads on encapsulated hASCs. The use of stem cells, has been exploited to overcome the limitations associated to tissue-specific cell sources, like difficulties in tissue harvesting and cell isolation, cell yields and limited proliferation. Human ASCs were selected as an adult stem cell source with interesting advantages over other commonly used stem cell populations (e.g. bone marrow mesenchymal stem cells), including, among other interesting features, higher proliferation rates, easier accessibility for tissue collection and increased cell numbers upon isolation [30].

Hydrogels based on PL have been increasingly investigated either employing selfcrosslinking mechanisms [17-19] or incorporating PL in hydrogel matrices [31-33]. Altogether, existing studies have been demonstrating a sustained release of endogenous biomolecules from PL-based hydrogels, resulting in a bioactive functionality and consequent modulation of cell behavior (e.g., stem cell differentiation, endothelial cell organization, etc.). Considering these remarkable aspects, we incorporated the biofunctionality of PL to advance our previously developed system based on ALG:GelMA hydrogel coating of silk suture threads [13]. In previously developed CLF, mechanical performance would be provided by the core fiber and the system would provide support to encapsulated cells. In ALG:GelMA hydrogels, although GelMA contains cell-binding motifs, like RGD sequences, and MMP-sensitive degradation sites [13] that are not present in ALG, the introduction of methcacryloyl moieties represents an artificial matrix lacking adequate biofunctionality. In this study, we proposed a PL hydrogel coating simultaneoulsy as an ECM-biomimetic fibrillar structure and a cocktail of biomolecules to encapsulate hASCs. 
Herein, the fabrication process of coating suture threads with PL hydrogel was first optimized. For this purpose, suture threads were initially embedded in an activation solution containing thrombin and $\mathrm{CaCl}_{2}$. Different incubation times were tested to evaluate their influence on the formation of PL hydrogel coating. Subsequently, suture threads were immersed in PL solution and incubated for 2 hours at $37^{\circ} \mathrm{C}$, as previously described [19]. The presence of these clotting factors induces a rapid selfassembly of PL into a 3D hydrogel [17]. In preliminary studies, a short incubation period of 5 minutes in thrombin/ $/ \mathrm{CaCl}_{2}$ solution and a subsequent immersion of sutures in PL immediately led to the formation of a PL hydrogel coating (Figure S1). Nonetheless, this resulted in a heterogeneous hydrogel distribution along the suture thread. Furthermore, this incubation step also influenced the thickness of the hydrogel coating. Longer incubation times resulted in the formation of a thicker, more homogenous and stable PL hydrogel layer. Hence, a 90-minute incubation period in thrombin/ $\mathrm{CaCl}_{2}$ solution was selected for the development of biofunctional composite living fibers. Cell encapsulation resulted in a well-distributed layer of PL-hASCs hydrogel, covering the suture thread/similarly/to previously reported coating processes $[11,13]$. Encapsulated hASCs were viable immediately after fabrication and up to 14 days in culture. Strikingly, cells were able to remodel their microenvironment, as seen by the variation in PL-hASCs hydrogel layer thickness over time in culture, owing to the cell supportive properties of PL. PL hydrogel retraction has been described as a result of fibrin matrix contraction by pulling forces exerted by encapsulated cells [19]. The existence of different compartments within the developed system provides an additional support for cellular activities during culture, overcoming the limitations of extensive hydrogel retraction. Accordingly, highly elongated cell morphologies induced by contact guidance mechanisms were evident as soon as 7 days in culture and even after prolonged culture periods. Human ASCs aligned/following the topography of the microfilaments composing silk suture threads. Previously developed composite suture threads coated with ALG:GelMA hydrogel also supported the elongation and alignment of encapsulated hTDCs; due to the slower degradation of ALG:GelMA hydrogel, two cellular regions have been shown after 7 days in culture - round cells embedded within the hydrogel layer and elongated cells at the surface of the suture thread [13]. Alternatively, PL hydrogel represents an ECM mimetic fibrillar network that is rich in cell binding sites and molecules involved in cell adhesion [34], possibly enabling faster migration and 
microenvironment remodeling, in comparison to the previously developed ALG:GelMA hydrogel as seen by the presence of round hASCs within the hydrogel layer at day 7 (Figure S2). Notably, the (bio)chemical composition of the hydrogel coating can potentially influence the behavior of different cell types. Indeed, when encapsulating hASCs in our previously reported composite sutures, cells remained round within the ALG:GelMA hydrogel layer even after 7 days of culture, in comparison to hTDCs, which were already migrating and elongating at the surface of the core thread (Figure S2). Hence, this study also showed the potential of tailoring the outer compartment of composite living fibers, independently of the core thread, to impact the behavior of encapsulated cells.

Furthermore, hASCs encapsulated in PL hydrogel rapidly deposited a highly oriented collagen-rich matrix. Collagen type I is a key protein in the ECM of several tissues of the human body, including bone, skin, tendons and ligaments. Furthermore, collagen type III is involved in the regulation of collagen type I fibrillogenesis and in tissue repair through a temporospatial coordinated expression during healing phases [35, 36]. In this work, hASCs strongly expressed and deposited both collagen types, further suggesting a highly supportive role of PL to maintain cellular functions over time in culture. Moreover, encapsulated hASCs could establish connections between independent fibers inside the same culture well (Figure S3), as a result of the chemotactic properties described for PL and other platelet preparations [37, 38].

Additionally, composite living fibers have been reported to withstand handling and textile assembling $[11,13]$. The coating of suture threads with PL hydrogel enables a faster in vitro maturation of the developed construct through an accelerated hydrogel layer contraction, migration of encapsulated cells to the core fiber and rapid triggering of microenvironment remodeling. Thus, PL-hASCs hydrogel coated suture threads may be used as living sutures aiming at potentially enhancing tissue repair or further assembled to generate living scaffolds.

\section{Conclusion}

In this work, we explored the concept of composite living fibers using suture threads coated with a hydrogel layer based on platelet lysates. Developed PL-hASCs hydrogel-coated suture threads represent a simple multi-compartment and multifunctional system. Core suture threads can provide the mechanical compliance required at the tissue level, whereas PL hydrogel, as a cocktail of growth factors and 
other biomolecules, offers biofunctionality to guide the biological activities of encapsulated cells and, possibly, recruited cells in vivo. Altogether, our results represent a promising strategy to be explored either as living sutures toward enhancing tissue repair, ultimately guiding regeneration or as living scaffolds once assembled by textile techniques.

\section{Acknowledgements}

Work developed under the framework of the Cooperation Agreement established with the Serviço de Imuno-Hemoterapia do Centro Hospitalar de S. João, EPE. The authors would like to thank to the Plastic Surgery Department of Hospital da Prelada (Porto, Portugal) for providing lipoaspirate tissue samples. Authors acknowledge the financial support from the ERC Grant CoG MagTendon nr 772817; and the Project NORTE-01-0145-FEDER-000021 (RCA pos-doc grant): "Accelerating tissue engineering and personalized medicine discoveries by the integration of key enabling nanotechnologies, marine-derived biomaterials and stem cells", supported by Norte Portugal Regional Operational Programme (NORTE 2020), under the PORTUGAL 2020 Partnership Agreement, through the European Regional Development Fund (ERDF); FCT-Fundação para a Ciência e a Tecnologia for the $\mathrm{PhD}$ grant of IC (PD/BD/128088/2016) and pos-doc grant of RMAD (SFRH/BPD/112459/2015); and HORIZON 2020 under the TEAMING/Grant agreement No 739572 - The Discoveries CTR. R.A. was the recipient of a scholarship from the School of Engineering of the University of Bologna (Italy).

\section{Conflicts of interest}

The authors declare no conflicts of interest.

\section{References}

1. Rajeswari, R. et al. (2015) Elastomeric core/shell nanofibrous cardiac patch as a biomimetic support for infarcted porcine myocardium. Tissue Engineering Part A 21 (7-8), 1288-1298.

2. Wu, S. et al. (2017) Living nanofiber yarn-based woven biotextiles for tendon tissue engineering using cell tri-culture and mechanical stimulation. Acta Biomater 62, 102-115.

3. Laranjeira, M. et al. (2017) 3D mimicry of native tissue fiber architecture guides tendon-derived cells and adipose stem cells into artificial tendon constructs. Small DOI; 10.1002/smll.201700689. 
4. Cooper, J.A. et al. (2007) Biomimetic tissue-engineered anterior cruciate ligament replacement. Proc Natl Acad Sci U S A 104 (9), 3049-3054.

5. Chen, M.-C. et al. (2013) Electrically conductive nanofibers with highly oriented structures and their potential application in skeletal muscle tissue engineering. Acta Biomater 9 (3), 5562-5572.

6. Zhang, J. et al. (2014) Wet-spun poly(e-caprolactone) microfiber scaffolds for oriented growth and infiltration of smooth muscle cells. Mater Lett 132, 59-62.

7. Onoe, H. et al. (2013) Metre-long cell-laden microfibres exhibit tissue morphologies and functions. Nature Materials (12), 584-590.

8. Kang, E. et al. (2011) Digitally tunable physicochemical coding of material composition and topography in continuous microfibres. Nature Materials 10, 877-883.

9. Costa-Almeida, R. et al. (2017) Multicomponent hydrogel fibers for tendon tissue engineering: combining polyelectrolyte complexation and microfluidics. ACS Biomaterials Science \& Engineering 3, 1322-1331.

10. Sant, S. et al. (2017) Self-assembled Hydrogel Fiber Bundles from Oppositely Charged Polyelectrolytes Mimic Micro-/nanoscale Hierarchy of Collagen. Adv Funct Mater 27, 1606273.

11. Akbari, M. et al. (2014) Composite living fibers for creating tissue constructs using textie techniques. Adv Funct Mater 24 (26), 4060-4067.

12. Tamayol, A. et al. (2016) Textile tissue engineering: a path towards organ weaving. Frontiers in Bioengineering and Biotechnology Conference Abstract: 10th World Biomaterials Congress. doi: 10.3389/conf.FBIOE.2016.01.00108.

13. Costa-Almeida, R. et al. (2018) Cell-laden composite suture threads for repairing damaged tendons. J Tissue Eng Regen Med 12, 1039-1048.

14. Anitua, E. et al. (2004) Autologous platelets as a source of proteins for healing and tissue regeneration. Thromb Haemost 91, 4-15.

15. Crespo-Diaz, R. et al. (2011) Platelet lysate consisting of a natural repair proteome supports human mesenchymal stem cell proliferation and chromosomal stability. Cell Transplant 20, 797-811.

16. Mendes, B.B. et al. (2018) Blood derivatives awaken in regenerative medicine strategies to modulate wound healing. Adv Drug Del Rev 12, 376-393.

17. Robinson, S.T. et al. (2016) A novel platelet lysate hydrogel for endothelial cell and mesenchymal stem cell-directed neovascularization. Acta Biomater 36, 86-98.

18. Fortunato, T.M. et al. (2016) Platelet lysate gel and endothelial progenitors stimulate microvascular network formation in vitro: tissue engineering applications. Sci Rep 6, 25326.

19. Mendes, B.B. et al. (2018) Human-based fibrillar nanocomposite hydrogels as bioinstructive matrices to tune stem cell behavior. Nanoscale 10, 17388-17401.

20. Silva, E.D. et al. (2017) Multifunctional magnetic-responsive hydrogels to engineer tendon-to-bone interface. Nanomedicine 14, 2375-2385.

21. Ito, R. et al. (2013) Efficacy of the controlled release of concentrated platelet lysate from a collagen/gelatin scaffold for dermis-like tissue regeneration. Tissue Engineering Part A 19, 1398-1405.

22. Sandri, G. et al. (2014) Platelet lysate embedded scaffolds for skin regeneration. Expert Opinion on Drug Delivery 12, 525-545.

23. Rossi, S. et al. (2013) "Sponge-like" dressings based on biopolymers for the delivery of platelet lysate to skin chronic wounds. Int J Pharm 440, 207-215.

24. Babo, P. et al. (2014) Platelet lysate membranes as new autologous templates for tissue engineering applications. Inflammation and Regeneration 34, 33-44. 
25. Costa-Almeida, R. et al. (2018) The effects of platelet lysate patches on the activity of tendon-derived cells. Acta Biomater 68, 29-40.

26. Santo, V.E. et al. (2012) Chitosan-chondroitin sulphate nanoparticles for controlled delivery of platelet lysates in bone regenerative medicine. J Tissue Eng Regen Med 6 Suppl 3, S47-S59.

27. Carvalho, P. et al. (2011) The effect of storage time on adipose-derived stem cell recovery from human lipoaspirates. Cells Tissues Organs 194, 494-500.

28. Gonçalves, A.I. et al. (2013) Understanding the role of growth factors in modulating stem cell tenogenesis. PLoS One 8 (12), e83734.

29. Ayres, C.E. et al. (2008) Measuring fiber alignment in electrospun scaffolds: a user's guide to the 2D fast Fourier transform approach. J Biomater Sci Polym Ed 19, 603-621.

30. Argentati, C. et al. (2018) Adipose Stem Cell Translational Applications: From Bench-to-Bedside. International Journal of Molecular Sciences 19, 3475.

31. Babo, P.S. et al. (2017) Platelet lysate-loaded photocrosslinkable hyaluronic acid hydrogels for periodontal endogenous regenerative technology. ACS Biomaterials Science \& Engineering 3, 1359-1369.

32. Neves, L.S. et al. (2017) Injectable hyaluronic acid hydrogels enriched with platelet lysate as a cryostable off-the-shelf system for cell-based therapies. Regenerative Engineering and Translational Medicine 3, 53-69.

33. Santo, V.E. et al. (2016) Engineering enriched microenvironments with gradients of platelet lysate in hydrogel fibers. Biomacromolecules 17, 1985-1997.

34. Brown, A.C. and Barker, T.H. (2014) Fibrin-based biomaterials: Modulation of macroscopic properties through rational design at the molecular level. Acta Biomater $10,1502-1514$.

35. Liu, X. et al. (1997) Type III collagen is crucial for collagen I fibrillogenesis and for normal cardiovascular development. Proceedings of the National Academy of Sciences of USA 94, 1852-1856.

36. Miedel, E.L. et al. (2015) Type III collagen modulates fracture callus bone formation and early remodeling. J Orthop Res 33, 675-684.

37. Ranzato, E. et al. (2009) Platelet lysate promotes in vitro wound scratch closure of human dermal fibroblasts: different roles of cell calcium, P38, ERK and PI3K/AKT. J Cell Mol Med 13, 2030-2038.

38. Castelnovo, L. et al. (2000) Human platelet suspension stimulates porcine retinal glial proliferation and migration in vitro. Invest Ophthalmol Vis Sci 41, 601-609. 